\title{
Mechanisms for Age-Related Changes of Fingertip Forces during Precision Gripping and Lifting in Adults
}

\author{
Kelly J. Cole, Diane L. Rotella, and John G. Harper \\ Department of Exercise Science, The University of lowa, lowa City, lowa 52242
}

\begin{abstract}
We investigated changes across the adult life span of the fingertip forces used to grip and lift objects and their possible causes. Grip force, relative safety margin (grip force exceeding the minimum to avoid slip, as a fraction of slip force), and skin slipperiness increased beginning at age 50 years. Skin slipperiness explained relative safety margin increases until age 60 years. Hence, after age 60 years, additional factors must elevate grip force. We argue that one factor is impaired cutaneous afferent encoding of skin-object frictional properties on the basis of three findings. First, only subjects 60 years and older increased their relative safety margins when the friction of the gripped surfaces was varied randomly versus experiments that varied only object weight. Skin slipperiness did not account for this behavior. Second, these older subjects scaled the initial portion of their force trajectories for the slippery surface during
\end{abstract}

experiments when friction was varied. Third, their grip force adjustments to new surfaces were delayed $\sim 100$ msec as compared with young subjects. Previous research has demonstrated that friction is signaled locally by fast-adapting afferents (FA I afferents), which decrease in number during old age. By contrast, adjustments triggered by object set-down, an event encoded by FA II afferents throughout the hand and wrist, were not delayed in our old subjects. Other findings included that anticipatory control of fingertip forces using memory of object weight was unimpaired in old age. Finally, old and young adults modulated their fingertip forces with equal smoothness and with similar relative intertrial variability.

Key words: human; prehension; motor control; grasp; hand; cutaneous; age; old; sensory
Reduced manual dexterity occurs in many healthy elderly persons, often affecting their quality of life and capacity for independent living (Jebsen et al., 1969; Potvin et al., 1980; Williams et al., 1982; Pacaud and Welford, 1989; Jette et al., 1990; Falconer et al., 1991; Hackel et al., 1992). Age-related changes of manual function are manifest in the fingertip forces used to grip and lift objects. Old adults' grip forces and "safety margins" (grip force in excess of the minimum grip force to prevent slip) averaged twice that of young adults (Cole, 1991; Kinoshita and Francis, 1996), and they demonstrated greater delays between grasping and lifting an object (Kinoshita and Francis, 1996). These behaviors are consistent with, but do not prove, cutaneous sensory impairment (see Johansson, 1996). Old adults can maintain a stable static force during pinch (Cole and Beck, 1994) or during isometric abduction of the index finger (Keen et al., 1994), but anecdotal evidence indicates that they modulate their grip and lift forces less smoothly than young adults (Cole, 1991; Kinoshita and Francis, 1996).

We do not understand completely the mechanisms underlying these age-related changes in fingertip force behavior. The greater safety margins may result from more slippery skin as we age (Cole, 1991; Kinoshita and Francis, 1996), but too few subjects have been studied to characterize these relationships accurately over the adult life span. Well known deterioration of cutaneous sensory functioning (see Kenshalo, 1986; Schmidt et al., 1990) may contribute to altered fingertip force control, but we lack

\footnotetext{
Received Oct. 26, 1998; revised Dec. 21, 1998; accepted Feb. 3, 1999.

This work was supported by National Institutes of Health, National Institute on Aging, Grant R01 AG12557. We thank Katrina Cannon for assistance with data reduction and analysis.

Correspondence should be addressed to Dr. Kelly J. Cole, Department of Exercise Science, S. 501 Field House, The University of Iowa, Iowa City, IA 52242. Copyright (C) 1999 Society for Neuroscience 0270-6474/99/193238-10\$05.00/0
}

direct evidence to support this theory. Reduced tactile sensory function in old age appears to be functionally unimportant for some manual tasks (Cole et al., 1998). Studies of visually guided arm movement indicate that older adults use sensory information from moment to moment to compensate for impaired feedforward controllers (Warabi et al., 1986; Morgan et al., 1994; Pohl et al., 1996; Yan et al., 1998); however, see Carnahan et al. (1998). Similarly, Kinoshita and Francis (1996) reported that adults $>80$ years old tend to disregard memory about an the mechanical properties of an object obtained from previous lifts when programming their fingertip forces.

We examined the fingertip forces that young, middle-aged, and old adults used to lift an object that we surreptitiously varied in weight or frictional properties at the grip surfaces (Westling and Johansson, 1984). We investigated (1) the relationships between age-related changes in skin friction and fingertip forces, (2) the capacity for tactile information to influence fingertip forces, (3) the capacity to program fingertip forces using memory for object weight and friction information gained from the previous lift, and (4) how smoothly subjects modulated their fingertip forces.

The weight and friction of the test object were varied in separate experiments. Therefore, we also examined (5) the effects of these different contexts on fingertip forces. If slippery objects are problematic for older adults, they may prevent object slips by strategically biasing their forces upward whenever they are likely to encounter objects of varying frictional properties.

\section{MATERIALS AND METHODS}

Subjects. Sixty-six healthy adults (age 22-86 years; 44 females and 22 males) participated in experiments during which we surreptitiously varied the friction of the object at the grip contact surfaces in one experiment ("Friction" experiment) and weight in another experiment ("Weight" experiment). Both experiments were performed during the 
same laboratory session and were repeated on a second day, typically within 1 week of the first session. We assigned the participants to the following analysis categories: Group I (ages 22-44 years, mean 32.7; 10 females and 5 males), Group II (ages 48-58, mean 54; 8 females and 4 males), Group III (ages 60-69, mean 65.7; 13 females and 4 males) and Group IV (ages 71-86, mean 77.1; 13 females and 9 males). All except two subjects chose to perform the experiments with their right hand. Informed consent was obtained from all subjects, and the study was conducted in accordance with the Declaration of Helsinki.

All participants were ambulatory, lived independently in the general community or in local retirement communities, and claimed to perform activities of daily living with little or no difficulty. Subjects claimed to be healthy but nevertheless reported various medical conditions prevalent among older adults: hypertension ( $n=15$, age 55-85 years), mild arthritis involving the hands $(n=25$, age $44-86$ years), heart disease $(n=6$, age $66-86$ years), and adult-onset diabetes $(n=2$, age 63 and 85 years). Four subjects (age 67-85 years) reported a history of carpal tunnel syndrome, with two having undergone successful surgical intervention several years ago. All four subjects reported no current symptoms consistent with carpal tunnel syndrome and did not show positive results to Phalen's test or Tinel's sign. Nine participants without a history of carpal tunnel syndrome or other peripheral neuropathy exhibited positive Phalen's test or Tinel's sign but claimed to experience no pain, numbness, tingling, or loss of feeling in their arm or hand during the experiments. Five subjects (age 66-71 years) suffered transient ischemic attacks more than six years previously but reported full recovery without recurrent signs or symptoms.

All participants appeared to be alert and cognizant of their surroundings. The "Mini-Mental State" examination (Folstein et al., 1975) was administered and yielded passing scores (25-30) in all subjects. Individuals with borderline passing scores of 25-27 (ages 57-69 years; 6 males, 5 females) responded correctly to two current event questions (e.g., Who is the President of the United States?).

Tactile pressure thresholds were obtained from the distal volar pads of the thumb and index finger of subjects by using Semmes-Weinstein pressure filaments (Smith and Nephew Roland, Menominee Falls, WI). We used a descending method of limits to establish a threshold. The finger and thumb each were tested approximately midway between the center of the pad and the radial margin of the finger and ulnar margin of the thumb, respectively. We attempted to contact precisely the same skin site on each filament application. A threshold was recorded for the smallest filament diameter (buckling force in $\log _{10} 0.1 \mathrm{mg}$, according to the manufacturer's calibration) that could be perceived on at least $70 \%$ of its applications.

Apparatus. The object that subjects grasped and lifted (Fig. 1A) has been described by Eliasson and colleagues (1995). The gripping surfaces were two opposing Plexiglas plates $(35 \times 35 \mathrm{~mm})$ that were parallel to each other and to the vertical plane, with a separation of $2.2 \mathrm{~cm}$ between the digit contact surfaces. These exchangeable plates were covered with black sandpaper (\#320 aluminum oxide) or a slippery black acetate fabric. A hidden cavity in the base of the object accepted exchangeable masses that allowed us to vary the object's weight. Load cells transduced the normal ("grip") force separately at both grip surfaces and vertical tangential (load) force at both grip surfaces. An accelerometer transduced the vertical acceleration of the test object. The lift-off of the object from the table was detected with a circuit that included a metal plate in the object's base that was grounded when in contact with a plate located on the table. An ultrasonic position transducer allowed us to monitor vertical position.

Procedures. Subjects washed their preferred hand with a mixture of lemon juice and water and dried it. Their arm hung vertically with the forearm, wrist, and hand projected anteriorly in intermediate pronosupination. They sat alongside a small table $(91 \times 61 \mathrm{~cm})$, with the table surface slightly lower than elbow level. Subjects were instructed to grasp the object approximately at the centers of the gripping surfaces by using a precision grip of the index finger and thumb, to lift the object vertically a few inches, and then to view a clock positioned directly in from of them. They were to hold the object stationary for $\sim 4 \mathrm{sec}$ before returning it to the table. Subjects achieved the lifting action mainly via increased elbow flexion, with the forearm and elbow unsupported.

On approximately one of every four trials the subjects were instructed to lift the object to a stationary position and then, after a few seconds, slowly to relax their grip force to allow the object to slip between their fingers. This procedure typically produced from one to several discrete slips of the object until it eventually fell from grasp, landing onto a foam rubber cushion (Fig. 1B). These slips were used to estimate the slip force (normal force at which slip began) and the coefficient of static friction at each digit (see Data Analysis, below).

In the Friction experiment the "slipperiness" of the object [4 newton (N) weight] was varied across trials by using either acetate or sandpaper coverings at the two grasp surfaces (Johansson and Westling, 1984; Cadoret and Smith, 1996). Subjects first lifted the object with the sandpaper-covered surfaces for 10 consecutive lifts. We then varied the surface material (in pairs) on subsequent trials, using the same order for all subjects until we acquired 26 trials with sandpaper and 19 trials with acetate (excluding "slip" trials). This procedure yielded six trials when the acetate surface preceded sandpaper (A-S), five trials when sandpaper preceded acetate (S-A), five trials when sandpaper preceded sandpaper (S-S), and eight trials when acetate preceded acetate (A-A). The two gripping surfaces were removed after every trial. Low levels of ambient illumination prevented the subjects from visually discriminating the surface materials.

In the Weight experiment we surreptitiously varied object mass to achieve weights of 2 or $4 \mathrm{~N}$ and used sandpaper to cover the gripping surfaces. Subjects performed 31 lifts $(15$ at $4 \mathrm{~N}$ and 16 at $2 \mathrm{~N}$ object weights, excluding slip trials). There were seven trials when the $4 \mathrm{~N}$ object preceded the $2 \mathrm{~N}$ object (4-2), seven trials when the $2 \mathrm{~N}$ object preceded the $4 \mathrm{~N}$ object (2-4), four trials with the $4 \mathrm{~N}$ object preceded the $4 \mathrm{~N}$ object (4-4), and six trials with the $2 \mathrm{~N}$ object preceded the $2 \mathrm{~N}$ object (2-2).

Data analysis. Data were acquired and analyzed with a personal computer running SC/ZOOM software (Department of Physiology, Umeå University, Umeå, Sweden). All signals were sampled with 12-bit resolution. The grip and load forces at both digits and the acceleration signal (root mean-square-processed, rise and decay time constants of 1 and $3 \mathrm{msec}$ ) were sampled at 400 samples/sec. Vertical position and contact signals were collected at 100 samples/sec. The mean normal force (hereafter "grip force") was calculated at each digitized point: (nor$\mathrm{mal}_{\text {finger }}+$ normal $_{\text {thumb }}$ )/2. The total vertical tangential load force (hereafter "load force") was determined by summing the vertical tangential load force at both digits. Grip and load force rates were derived by using a symmetrical \pm 3 point numerical time differentiation.

Torque loads around the axis perpendicular to the grip surfaces were not measured. Such loads can occur when the grasping digits do not contact the object in vertical alignment with the center of mass and can cause higher grip forces as subjects attempt to prevent pitch rotation of the object (Kinoshita et al., 1997). As a result, we could not assess the extent to which variations in grip force reflect off-center fingertip placement.

Sequential preload and load phases were defined for each lift (Johansson and Cole, 1994). The preload phase (Fig. 1C, interval $a$ ) was measured separately for each digit; it began with any increase in grip force rate for that digit greater than background noise levels and ended with the start of the increase in lift force rate for that digit. The load phase (interval $b$ ) commenced with a sustained increase in lift force rate and ended with the lift-off of the object from the support surface, as indicated by the contact signal. We also determined when the test object was replaced to the support surface by using the contact signal and when the grip force began its sharp decrease (reflected in the grip force rate signal) after the object's contact with the support surface (interval $c$ ). For most results we report the grip force and relative safety margin at the instant the object contacted the support surface ("set-down") to minimize the effects of acceleration-related variations in vertical load during the lifting phase. Unless noted otherwise, there were no meaningful differences between data measured at set-down and data obtained at lift-off from the support surface or $1.5 \mathrm{sec}$ later.

Trials with instructed slips provided data only for estimating the slip force (normal force slipping digit ) and the coefficient of static friction (load force slipping digit $_{\text {slip force }}$ slipping digit ) and were not included in other analyses of fingertip forces. Slip events were characterized by a sharp decline in the vertical load force at one digit synchronous with an increased vertical load at the other digit and vertical object motion indicated by the accelerometer signal (Fig. $1 B$ ). An estimate of each subject's coefficient of friction for each digit was calculated separately for each friction and weight condition by averaging the coefficients obtained across all respective slip events. However, because we measured only vertical tangential loads, the coefficient of friction will be underestimated if horizontal tangential loads exist or if torque loads exist at the digit around the axis perpendicular to the gripping surface (Kinoshita et al., 1997). With the $4 \mathrm{~N}$ weight the object's center of mass was aligned nearly vertically with the instructed fingertip placements (center of the grasp 

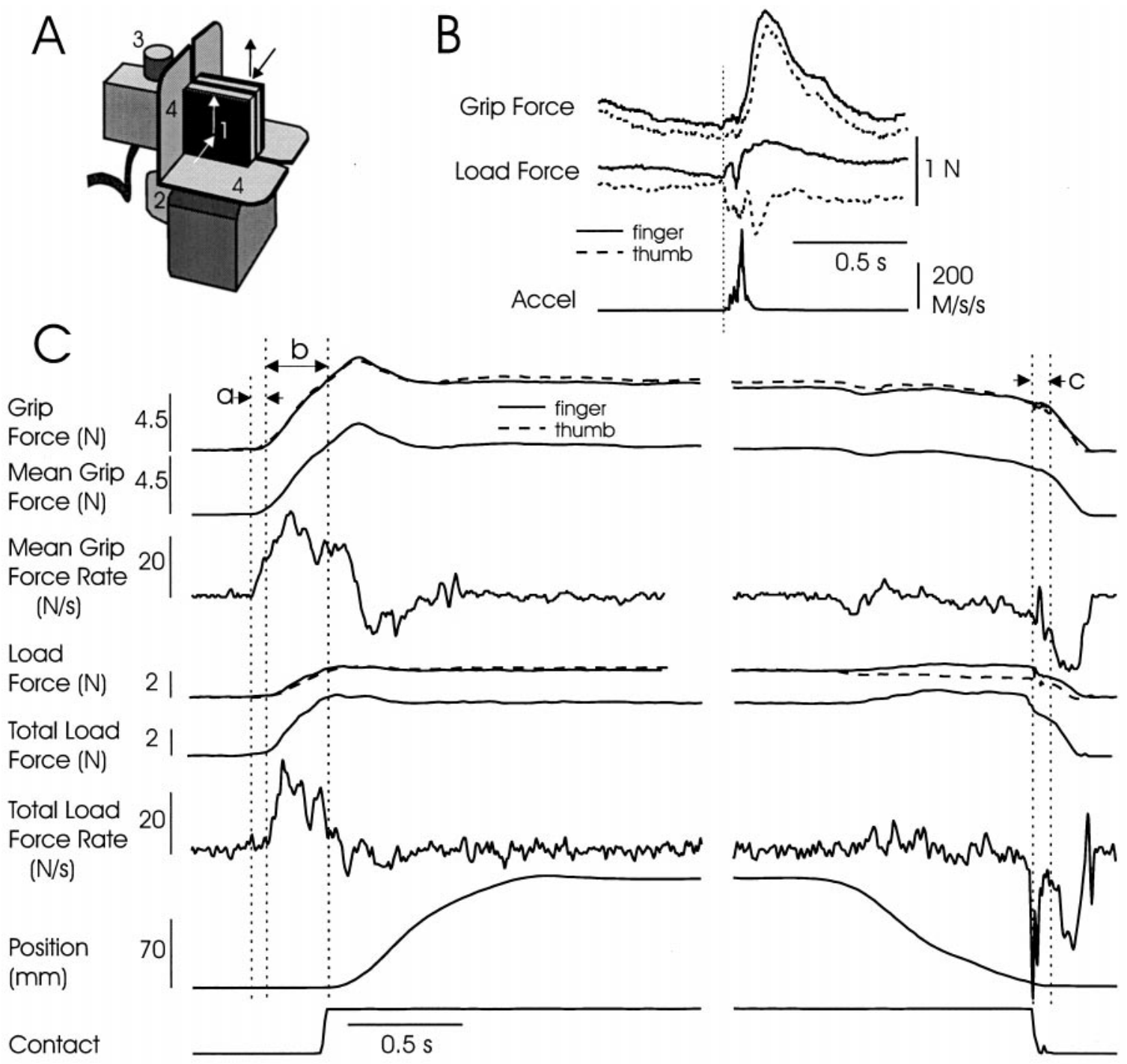

Figure 1. A, Instrumented object. 1, Exchangeable grip surface for thumb (grasp with right hand); grip surface for finger hidden from view. Load cells in the object transduced the forces normal (grip) and vertical tangential (load) to the surfaces separately for the finger and thumb. 2, Handle attached to exchangeable mass, not in subjects' view. 3, Ultrasonic transmitter for vertical position sensing. 4, Rigid shrouds to avoid finger and thumb pad placement on far edges of the grip surfaces. $B$, Slip at the thumb during a single trial, as indicated from object acceleration, precipitous unloading at the thumb grip surface, and increased load at the finger. Notice the slip-triggered increase in grip force $\sim 100$ msec after slip onset. The grip and load forces at the slipping surface on acceleration onset were used to estimate the inverse coefficient of friction. $C$, Examples of signals recorded during a single trial from a young adult and measurements taken for analysis. Interval $a$ (preload) began on first evidence of digit contact with either grip surface and ended with the rise in load force. Interval $b$ (loading) began on the rise in load force and ended on vertical motion of the object. Interval $c$ indicates the time from object set-down to the beginning of the precipitous fall in grip force that marked the onset of grasp release.

surface plates), but tangential torques existed for the $2 \mathrm{~N}$ weight with these fingertip placements. Tangential torques were assumed to dissipate primarily on the initial slip and accompanying pitch rotation of the object. Therefore, slip events that yielded extreme values within a subject's distribution of frictional coefficients (less than the 25 th percentile) were discarded.

We computed a "relative safety margin" at the index finger at various times during each trial (object lift-off, $1.5 \mathrm{sec}$ later, and at object setdown), which was the safety margin at the index finger (normal force finger $_{\text {}}$ - slip force finger $_{\text {) divided by the slip force }}$ finger.

Statistical testing of the data via ANOVA and ANCOVA used mean values obtained from each subject within an experiment and used a repeated-measures design (across age groups, within subjects). The mean values for each subject first were transformed (natural logarithm) to obtain normal data distributions for ANOVA and ANCOVA. Multivariate methods were used for more robust calculations of repeatedmeasures effects whenever there were three levels or more of the "withinsubjects" factor. In this case Rao's $R$ statistic was calculated, which is a transformed version of Wilk's lambda, and follows an $F$-distribution in most cases (Lindeman et al., 1980). Post hoc testing used Tukey's Honestly Significant Difference for unequal sample size. All statistical calculations were performed with STATISTICA software (Version 5, StatSoft, Tulsa, OK). Values in graphs and text are medians (graphs include 25th and 75th percentiles), unless otherwise noted, and were calculated from data before logarithmic transformation. Results report data from the second session of each experiment, unless otherwise noted. Statistical comparisons of the two sessions revealed few differences for each subject or age group and support identical conclusions across sessions.

\section{RESULTS}

The grip forces present at the end of the hold phase (set-down) in the Friction experiment (Fig. 2, top panel) were greater for the three older groups as compared with the youngest subject group $\left(F_{3,62}=7.07 ; p<0.0004\right.$ Age $\times$ Surface Interaction $)$ by $35-73 \%$ 

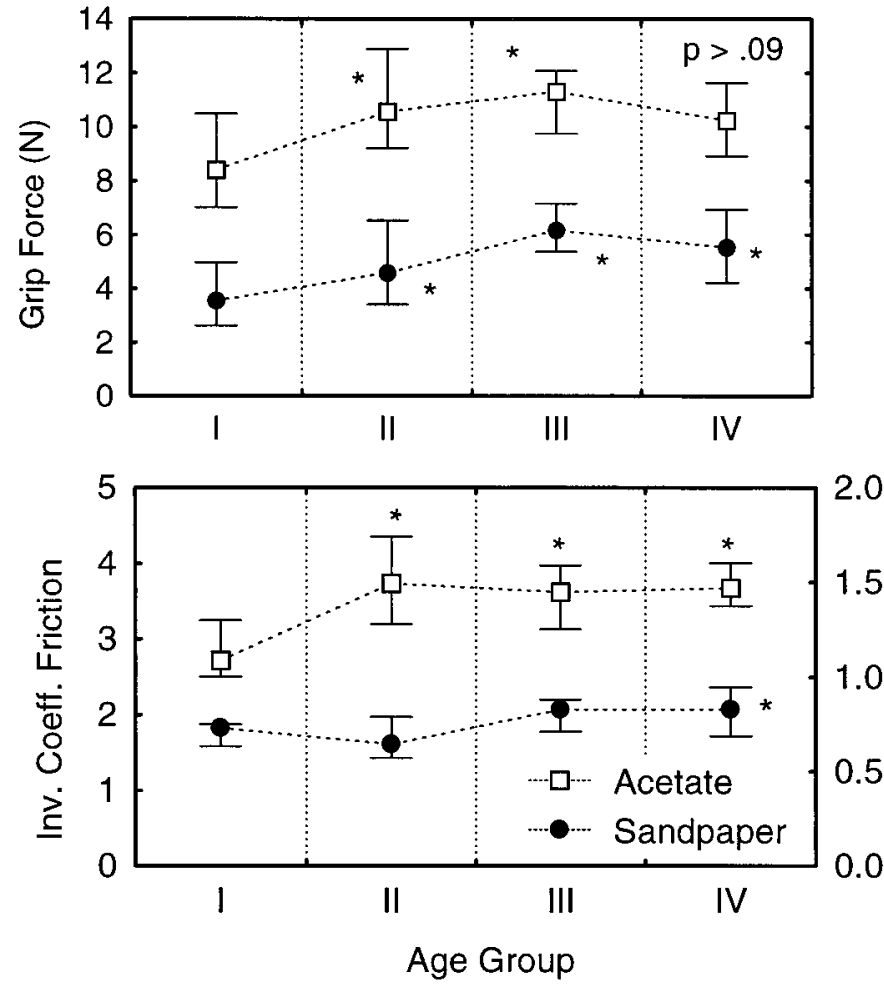

Figure 2. Top panel, Grip force (medians and whiskers indicate 25 th and 75th percentiles) at the moment of object set-down for each of the four age groups (Group I, 22-44 years; Group II, 48-58; Group III, 60-69; Group IV, 71-86). Measured are sandpaper grip surface ( filled symbols) and acetate fabric surface (open symbols). Post hoc testing within each surface revealed significant differences between Group I and each of the older groups (asterisks indicate $p<0.05$ ), except for acetate, Group IV $(p=0.09)$. Bottom panel, Inverse coefficient of friction at the index finger across age groups; shown are sandpaper ( filled symbols, right y-axis scale) and acetate (open symbols, left y-axis scale). Post hoc testing within the acetate surface revealed significant differences between Group I and each of the older groups, but for sandpaper only Group IV differed significantly from Group I.

for the sandpaper surface and by $18-32 \%$ for the acetate surface. We were interested first in how completely age-related increases in skin slipperiness, represented by measures of the inverse coefficient of friction, accounted for the greater grip forces in the old subjects. The inverse coefficient of friction increased with age but depended on the gripping surface (Fig. 2, bottom panel). The inverse coefficients for the acetate gripping surface were greater for the three older age groups as compared with the youngest group, whereas only the oldest group of subjects showed greater inverse coefficients for the sandpaper surface $\left(F_{3,59}=6.003 ; p<\right.$ 0.001 for Age $\times$ Surface Interaction). There were no statistically significant differences between men and women at any age for either surface.

These findings indicate that skin slipperiness can account only for some of the greater grip force that occurred with increasing age. To explore this further, we used the inverse coefficients of friction obtained with the sandpaper and acetate surfaces as changing covariates in an ANCOVA to test for differences in grip force (at set-down) across age groups. An interaction between age group and surface type was found $\left(F_{3,58}=9.31 ; p<0.0001\right)$. Therefore, separate one-way ANCOVAs were performed by using the data for each surface covering to adjust the grip forces for the effects of skin slipperiness. For the sandpaper surface, grip

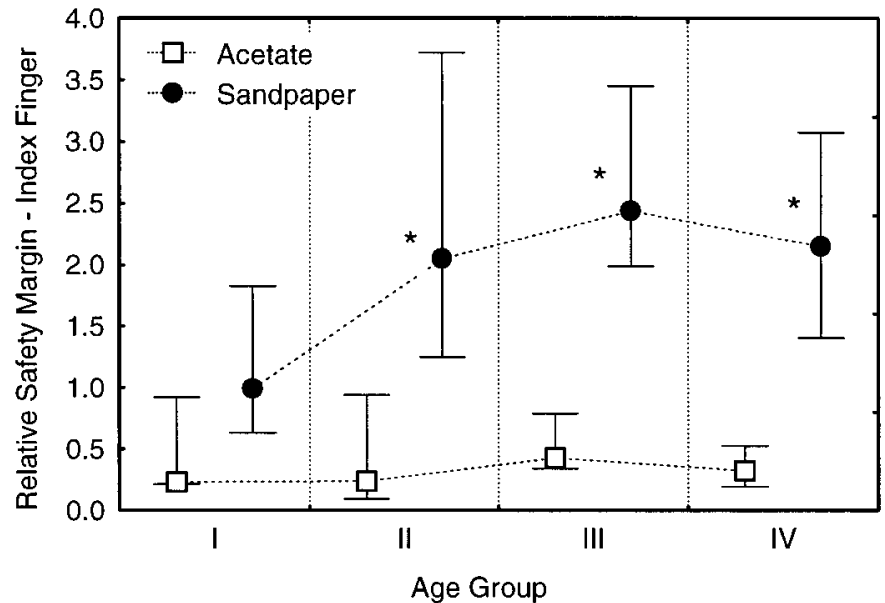

Figure 3. Relative safety margin (medians at 75 th and 25 th percentiles) at the index finger for acetate (open symbols) and sandpaper (filled symbols) surfaces across age groups. Post hoc testing (sandpaper) revealed significant differences between Group I and each older group (asterisks indicate $p<0.05)$.

forces for Groups III and IV were 33-57\% greater than those of Group I even after accounting for the correlation with skin slipperiness $(3.7,5.0,5.8$, and $5.0 \mathrm{~N}$, respectively, for Groups $\mathrm{I}-\mathrm{IV} ; F_{3,59}=4.95 ; p<0.004 . p<0.005$ for post hoc comparison of Groups III and IV with Group I, but $p>0.14$ for post hoc comparison of Group I with Group II). For the acetate surface mean grip force increased by only $4-15 \%$ in the old groups $(9.3$, $10.7,10.5$, and $9.7 \mathrm{~N}$, respectively, for Groups I-IV; $F_{3,59}=1.51$; $p>0.22$ ), which indicates that the older subjects' greater grip forces for the acetate surface mainly reflect adjustments for their more slippery skin.

Age-related changes in relative safety margin at the index finger were consistent with the preceding analyses of grip force and its correlation with skin slipperiness. Subjects in Groups II-IV used relative safety margins for the sandpaper surface that were twice as large as the relative safety margins that the young subjects used, but there were statistically unreliable increases of $12-33 \%$ for the acetate surface (Fig. $3 ; F_{3,58}=4.87 ; p<0.004$, Age $\times$ Surface Interaction). Depending on the age group, some or all of the age-related increases in relative safety margin for the sandpaper surface can be attributed to increasing skin slipperiness in view of results from the preceding ANCOVA. For example, larger relative safety margins in Group II can be attributed completely to their greater skin slipperiness as compared with Group I. This is because the increase in grip force for Group II correlated strongly with skin slipperiness but exceeded the amount needed to match the relative safety margins of Group I. However, this exaggerated frictional scaling cannot account for the $33-57 \%$ difference in grip force that remained between Groups III and IV and Group I after accounting for the covariance of grip force with the inverse coefficients of friction.

Reports of declining populations of Meissner's corpuscles in the fingertips of old adults and the role of these endings for encoding friction (Cauna, 1965; Johansson and Westling, 1987) make it plausible that subjects older than 60 years (i.e., Groups III and IV) used greater relative safety margins for sandpaper because adapting their fingertip forces to object friction was difficult. If so, they may use large grip forces for sandpaper in the Friction experiment because on each trial there was a possibility that the 
surface could be slippery. To address this, we compared the grip forces that the older subjects used during the Friction and Weight experiments (see Materials and Methods). Subjects in Groups III and IV consistently used greater grip forces (at set-down) in the Friction experiment as compared with the same object $(4 \mathrm{~N}$, sandpaper) lifted in the Weight experiment $\left(F_{3,62}=3.135 ; p<\right.$ 0.032 Age $\times$ Experiment Interaction). Average increases in grip force across experiments were 33 and $27 \%$ for Groups III and IV, respectively $(p<0.001)$, and yielded increases in relative safety margin of $41 \%$ for Group III and $73 \%$ for Group IV $(p<0.02$ for each). By contrast, grip force increased only 3 and $8 \%$ for Groups I and II ( $p>0.99$ and $p>0.37$, respectively). The size of the grip force difference between the Friction and Weight experiments in Groups III and IV showed a weak but positive correlation $(r=$ 0.24) with tactile perceptual thresholds (Semmes-Weinstein filament).

Despite the smaller grip forces used in the Weight experiment as compared with the Friction experiment, Groups III and IV used relative safety margins in the Weight experiment that were from 41 to $104 \%$ greater than those of the young group $\left(F_{3,58}=\right.$ 4.82; $p<0.005$; main effect of Age, no interaction; $p<0.001$ for relevant post hoc tests). However, there were no statistically reliable differences in grip force across age groups when the lightest $(2 \mathrm{~N})$ object was lifted.

The force bias between the Friction and Weight experiments observed for Groups III and IV cannot be explained by their more slippery skin as compared with Group I, because no bias was observed for Group II, although their skin was more slippery than Group I. To explore this further, we combined Group I with II and Group III with IV to produce two new subject groups for analysis ("young" and "old," respectively). The inverse coefficients of friction for the acetate surface were mostly between values of 2.5 and 4.5 ( $82 \%$ of the subjects in the new "young" group and $97 \%$ of the "old" group). Across this similar range of frictional coefficients for the two age groups, the relative change in grip force across the two experiments (expressed as a percentage) was greater, on average, for old versus young subjects (Fig. 4). Moreover, the slopes of the regression lines (least-squares) for the percentage of change in grip force as a function of friction were not significantly different from zero $(p>0.2$ for both groups). Therefore, an age-related factor(s) other than skin slipperiness must account for the difference.

We analyzed the fingertip forces used during trials that followed a change in surface covering for further evidence that old subjects had difficulties detecting changes in friction (surface covering) or problems in using this information promptly to adapt their fingertip forces. Ensemble averaged traces of the grip and lifting forces (Fig. 5), aligned with the initial rise in grip force rate, were computed for a randomly selected subset of subjects ( $n=11$ from $22-43$ years old; $n=21$ from $71-85$ years old). Trials were averaged within each subject according to the sequence of surface coverings: acetate trials that followed sandpaper trials (S-A; Fig. 5, left panels), sandpaper trials that followed acetate trials (A-S; Fig. 5, right panels), sandpaper trials that followed sandpaper trials (S-S), and acetate trials that followed acetate trials (A-A).

Many of the young subjects (64\%) increased their grip force during the initial loading phase at greater rates during A-A trials as compared with S-S trials, but fewer old subjects (26\%) exhibited this behavior. Instead, most of the old subjects $(74 \%)$ produced initial grip force rate trajectories that followed the trajectory for repeated lifts with the acetate surface $(\mathrm{A}-\mathrm{A})$ regardless of

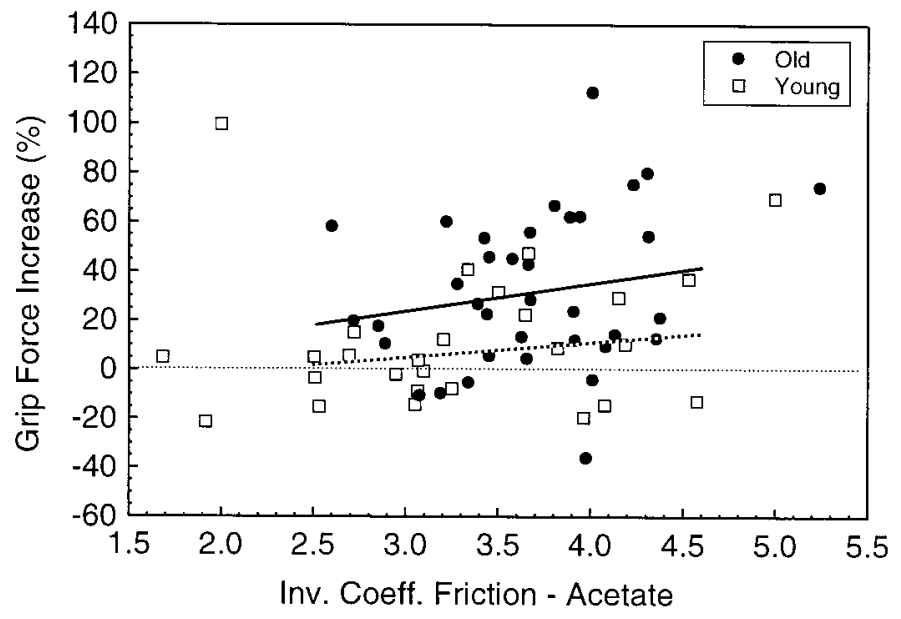

Figure 4. Average increase in grip force at object set-down (4 N object, sandpaper surface) for each subject in the "Friction" experiment as a percentage of grip force used in the "Weight" experiment. Data are plotted against each subject's inverse coefficient of friction at the index finger for the acetate surface. Groups I and II are combined (open symbols), and Groups III and IV are combined (filled symbols). Only trials that were preceded by a trial using the same object properties were included in the analysis (i.e., the second of two sequential trials with the sandpaper grip surfaces in the Friction experiment and likewise for the 4 $\mathrm{N}$ weight in the Weight experiment). Linear regression equations (leastsquares estimates) were fit across frictional coefficients between 2.5 and 4.5. Old group (solid line), Percentage Increase $=-13.5+12.2$. Inverse Coefficient of Friction. Young group (dotted line), Percentage Increase $=$ $-16.6+7.1 \cdot$ Inverse Coefficient of Friction

which surface was encountered on the preceding trial (Fig. 5, bottom panels). In effect, these old subjects scaled their grip force for the "worst-case" (most slippery) surface. This was true for all subjects over the age of 75 years (10 of 10 of the subset selected for ensemble average analysis) and for $36 \%$ of the young subjects. However, during the Weight experiment, when acetate surfaces were not encountered, old subjects decreased their grip force rates and clearly used different grip force trajectories for the $4 \mathrm{~N}$ (sandpaper) object during the two experiments (Fig. 5, bottom right panel; compare gray and dotted lines).

When most young subjects encountered a new surface (sandpaper, for example; Fig. 5, dashed traces in top right panel), the grip force and force rates over the first 100-200 msec followed trajectories that were similar to those used on repeated trials with the other surface (acetate, for example; Fig. 5, solid lines in top right panel). This is consistent with previous reports that we program our fingertip forces in accord with the mechanical properties of the object that were obtained during the preceding lift, unless we receive information before the lift (e.g., visual, haptic) concerning new object properties (for review, see Johansson, 1996). As noted previously, most old subjects used a force rate trajectory always scaled over the first few hundred milliseconds for the acetate surface. However, the grip force rate was modified appropriately during the loading phase by subjects of all ages (Fig. 5, triangles; compare solid and dashed lines). Specifically, these "on-line" modifications of grip force rate consisted of an increase if the new surface was acetate (for young subjects only, left panels) or a decrease if the new surface was sandpaper (for young and old subjects, right panels). The force-rate trajectory then followed the trajectory used on repeated trials with the new surface (Fig. 5; compare dashed and dotted lines).

The time required for individual subjects ( $71-85$ years, $n=21$; 

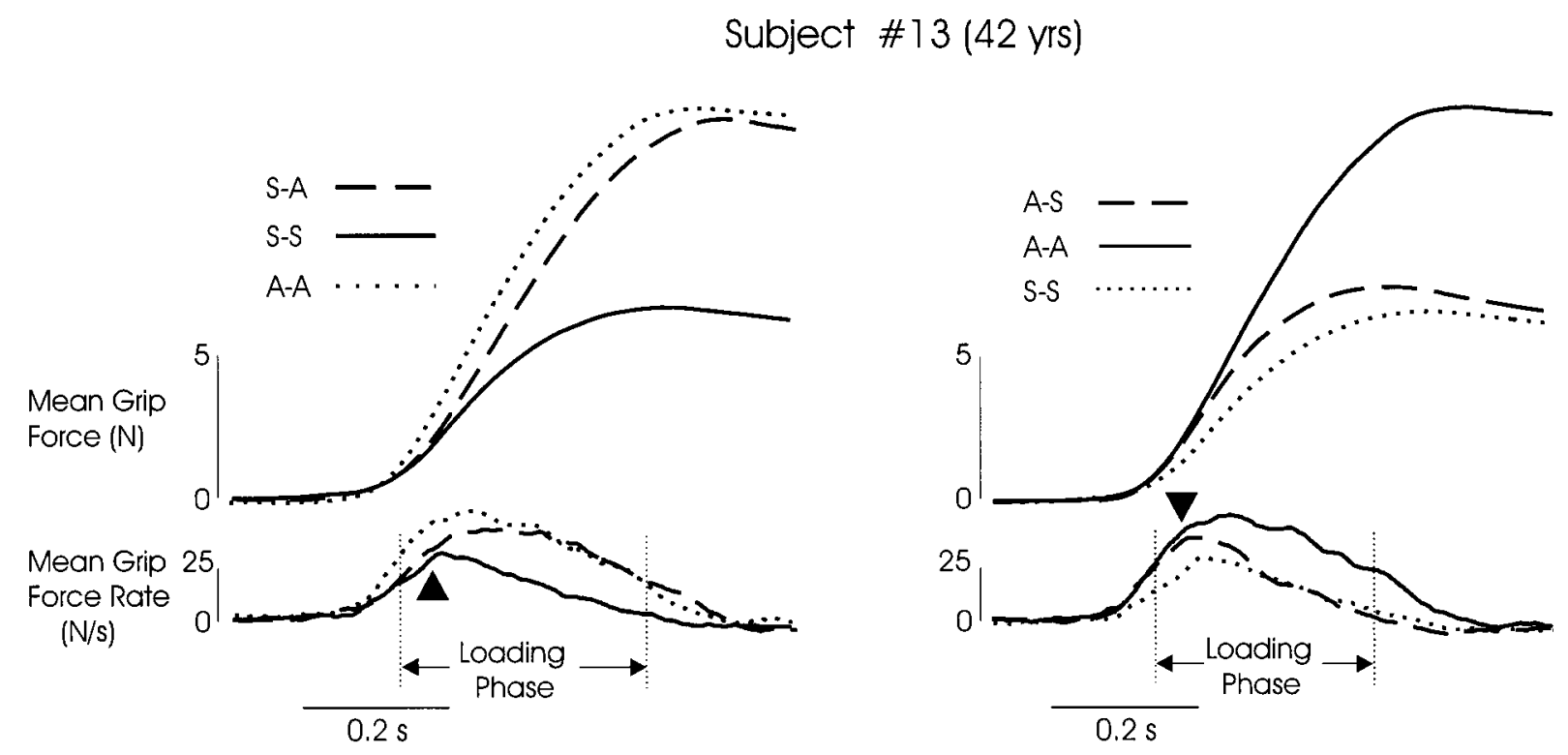

Subject \#57 (79 yrs)
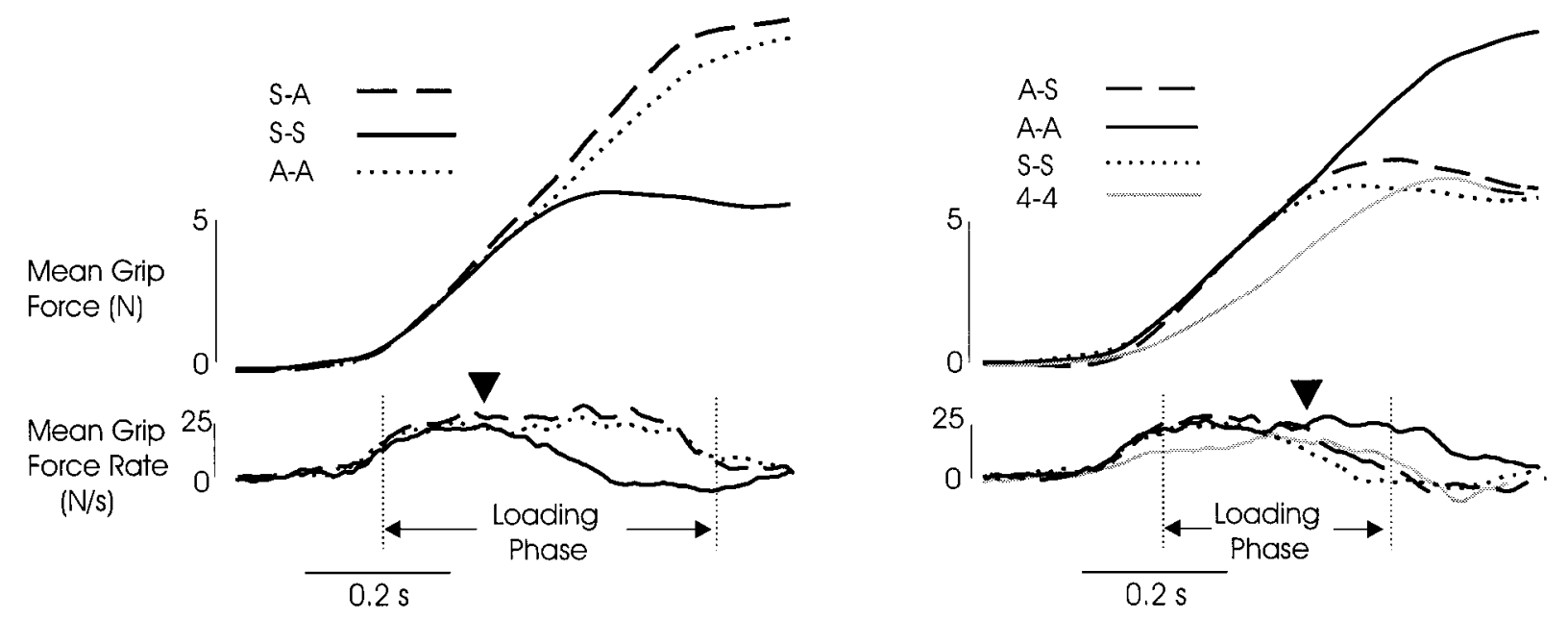

Figure 5. Ensemble averaged traces of grip force and grip force rate signals from a young adult (top panels) and an old adult (bottom panels). In each panel the dashed lines indicate trials with a new surface. Legends indicate the order of surface presentation, with the surface encountered on the preceding trial listed first and the surface of the current trial listed second (e.g., S-A is a trial with the acetate surfaces that followed a trial with the sandpaper surface). The gray lines in the bottom right panel represents data from the Weight experiment, in which lifts of the $4 \mathrm{~N}$ weight were presented sequentially. Vertical lines indicate the loading phase for the trials with the new surface. Filled triangles indicate the time at which the force rate trace representing the trial with the new surface (dashed line) diverged from the trace representing repeated trials with the other surface (solid line). Repeated trials with the new surface (dotted lines) are included and indicate that subjects adapted nearly completely to the new surface by the end of the loading phase (object lift-off).

22-43 years, $n=11$ ) to correct their grip force for the new surface was determined by measuring the time between the initial rise in grip force rates and the time at which the overlaid traces diverged (Fig. 5, triangles; compare solid and dashed lines). These response latencies for the old subjects were significantly longer than those for the young $\left(F_{1,26}=8.75 ; p<0.006\right.$, main effect of Age Group, Group by Surface Sequence, ANOVA), with the grip force correction occurring early during the loading phase for young subjects and later for older subjects. There was a large difference in latency between young and old subjects on A-S trials (110 and $205 \mathrm{msec}$, respectively; $p<0.02$, post hoc test) and on $\mathrm{S}-\mathrm{A}$ trials (110 and $160 \mathrm{msec}$, respectively; $p<0.019$, after eliminating four old subjects who showed response latencies of 0 msec and unusually long preload durations). However, the magnitude of these latency differences should be viewed with caution. The older adults' use of default force trajectories scaled for the acetate surface may yield overestimates of response latency, considering the measurement procedures we used.

We could discern no correspondence between a subject's response latencies and his/her health history or results of diagnostic testing of median nerve function. Also, response latencies were better explained by age group than by subjects' tactile perceptual thresholds (Semmes-Weinstein threshold; Fig. 6).

Despite the delayed adjustments in fingertip forces to new 

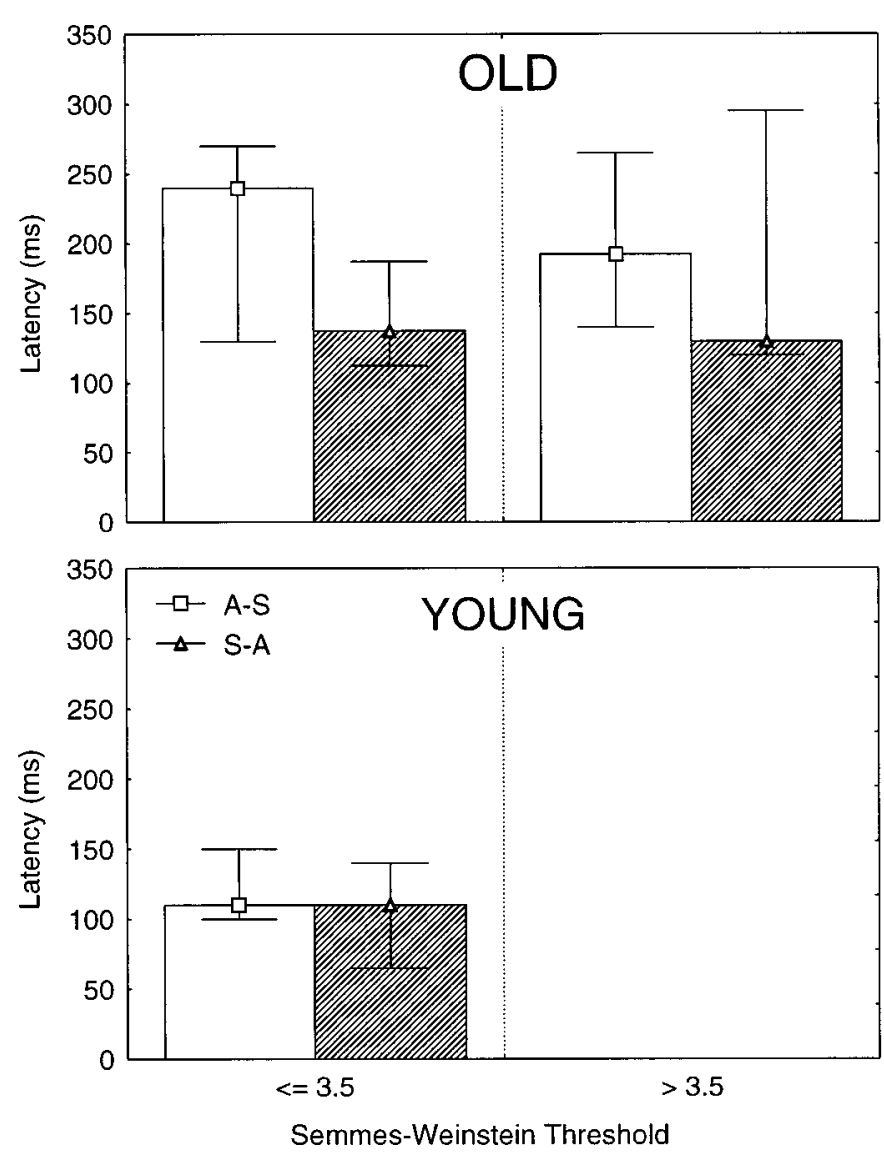

Figure 6. Latencies (medians at 75 th and 25 th percentiles) measured from the rise in mean grip force rate to the time that adjustments to the new grip surface appeared. Data are grouped according to the pressure sensibility threshold (Semmes-Weinstein filaments) obtained at the index finger. Top panel, Data from subjects older than 70 years. Bottom panel, Data from subjects younger than 48 years. Shown are trials with the sandpaper surface that followed acetate (open bars) and trials with acetate that followed sandpaper (hatched bars).

surfaces, all subject groups had adjusted their grip force appropriately to the new frictional properties before the object moved from the support surface (see Fig. 5; Rao $R_{3,60}=133.9 ; p<$ 0.0001 , Age Group $\times$ Surface Sequence, main effect of Surface Sequence). At object lift-off for the A-S category, all subject groups used grip forces that were only slightly greater than those for S-S trials ( $p>0.66$ for all age groups). Similarly, on S-A trials the subjects used grip forces that were not different from those for A-A trials ( $p>0.80$ for all groups).

Other mechanical events at the fingertip during the grasp and lift task appeared to trigger the required fingertip forces at similar latencies for young and old. For example, on returning the object to the support surface, contact with the table triggers a rapid decrease in grip force for the eventual release of the object (see Fig. $1 C$ ). The time from contact until the onset of this decrease in grip force did not differ across the age groups (78, 83, 71, and 75 msec for Groups I-IV, respectively; $F_{3,62}=0.447 ; p>0.72$, main effect of Age, Age $\times$ Surface ANOVA).

Inspection of grip and load force signals revealed other agerelated differences in the coordination between the grip and lifting forces. The older subjects demonstrated longer preload durations (time from the first evidence of object contact to sustained rise in lifting force; see Materials and Methods) than the youngest group $\left(F_{3,62}=2.7 ; p>0.053\right.$, main effect of Age; Age $\times$ Surface ANOVA, no interaction effect). Preload durations for the sandpaper surface were 140, 187, 238, and $288 \mathrm{msec}$ for Groups I-IV, respectively. Subjects did not attempt to lift the object during these extended preload phases but instead applied tangential forces downward, pressing the object against the table. These negative vertical tangential forces were more common and larger for the two oldest groups $(-0.18,-0.2,-0.31$, and $-0.4 \mathrm{~N}$ Groups I-IV, respectively; Rao $R_{3,60}=7.11 ; p<0.001$, main effect of Age, Age $\times$ Surface Sequence MANOVA).

Both old and young subjects programmed their fingertip forces in an anticipatory manner, based on the weight encountered during the previous lift. This was revealed by the size of the peak grip force rate during the loading phase when a $2 \mathrm{~N}$ object was lifted. Rates were greater when a $4 \mathrm{~N}$ object had been lifted on the preceding trial as compared with a $2 \mathrm{~N}$ object on the preceding trial (increase of 2.9, 4.0, 5.8, and $7.8 \mathrm{~N} / \mathrm{sec}$ for Groups I-IV, respectively; $F_{1,62}=55.29 ; p<0.0001$, main effect of Weight Sequence, Age $\times$ Weight Sequence ANOVA). Similar results were obtained on statistical testing of the peak lift force rate and lift force rate at lift-off. The greater increases in peak grip force rate for the older groups reflect an unexplained tendency toward faster grip and lift rates in the oldest subjects. There was no significant interaction effect between Age and Weight Sequence $(p>0.77)$.

We observed no clear evidence that older adults increased their grip force less smoothly than did young adults during the loading phase. Fourteen subjects (seven each from Groups I and IV) were selected randomly for inspection of their grip force and grip force rate traces. Five sequential trials were examined (4 $\mathrm{N}$ weight, sandpaper surface), taken from each subject's first experimental session before they experienced any variations in weight or grip surface material (Fig. 7). A young subject (\#7) showed the smoothest and, across trials, the most consistent force and force rate trajectories, whereas an old subject (\#63) showed trajectories that were least smooth and most inconsistent across trials. However, inspection of Figure 7 revealed no consistent trend in these characteristics across the remaining young and old subjects. Intertrial variability was analyzed quantitatively. Across all four age groups there were no differences in coefficients of variation (SD/ mean) during the Friction experiment for peak grip force $(0.288$, $0.295,0.281$, and 0.315 across groups for sandpaper and 0.206 , $0.207,0.207$ and 0.225 for acetate; $F_{3,62}=0.588 ; p>0.63$, main effect of Age Group, no interaction with Surface) or the grip force at lift-off $(0.221,0.280,0.237$, and 0.199 for sandpaper and $0.152,0.165,0.173$, and 0.157 for acetate; $F_{3,62}=1.22 ; p>0.31$ main effect of Age Group; significant interaction with Surface, but $p>0.48$ for all relevant across-age post hoc comparisons).

\section{DISCUSSION}

We confirmed reports of age-related increases in grip force and relative safety margin (Cole, 1991; Kinoshita and Francis, 1996). Consistent with Kinoshita and Francis (1996), large age-related increases in relative safety margin occurred for sandpaper surfaces, with smaller increases for acetate surfaces (after converting data in their Table 2 to relative safety margin). These increases began during the sixth decade (50 years) but were explained by increasing skin slipperiness only until age 60 years. We shall argue that declining cutaneous afferent function exacerbates safety margin increases after age 60 years.

Age-related increases in relative safety margin partly reflect the normal process of scaling the grip force for skin-object 

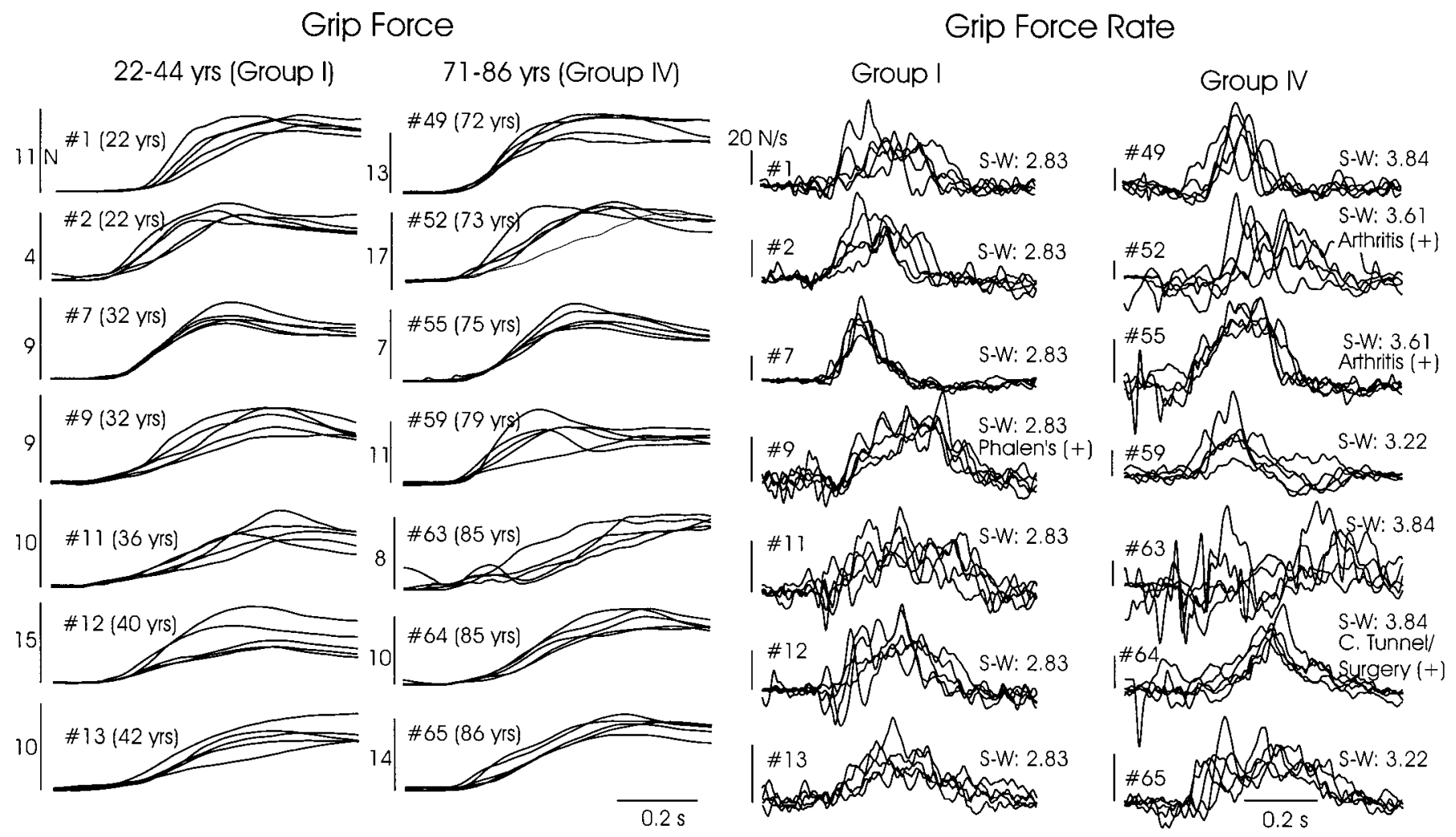

Figure 7. Grip force and grip force rate trajectories from five single trials per subject, superimposed within subject. Data are taken from the first session, sandpaper surface, beginning on the first trial after 10 practice lifts. Seven subjects each were selected randomly from Groups I and IV. Tactile sensibility threshold (Semmes-Weinstein filaments) at the finger is indicated for each subject, along with other positive findings from each subject's health history.

friction (Johansson and Westling, 1984; Cadoret and Smith, 1996). The large relative safety margins of our middle-aged subjects resulted solely from a grip force scaling factor that increased with increasing skin slipperiness, and not with age per se, confirming suggestions by Kinoshita and Francis (1996) and Kawai and colleagues (1995). This exaggerated frictional scaling helps to avoid slips of an object, which may be catastrophic when the coefficient of kinetic friction is low, because slipping objects will accelerate rapidly. Indeed, we observed that older adults often dropped the acetate-covered object during intentional slip trials after only a single slip (see Materials and Methods) but produced several slips per trial with the sandpaper covering.

Previous studies reported increased "skin slipperiness" in old adults for sandpaper and slippery surfaces like acetate but did not examine middle-aged subjects (Cole, 1991; Kinoshita and Francis, 1996). Our finding of increased slipperiness for acetate beginning at age 50 years, but for sandpaper only after age 70 years, is consistent with suggestions that the rough macrostructure of sandpaper dominates the frictional properties of the skin-object interface (Jenmalm and Johansson, 1997). Apparently, agerelated skin properties such as reduced hydration (Potts et al., 1984) contribute more to the coefficient of friction when smoother surfaces are handled. Therefore, age-related skin properties may not provoke excessive relative safety margins when individuals younger than 70 years handle rough surfaces (provided there is little chance of encountering a slippery object). This may be true particularly when lightweight objects are handled, based on the small effect of age when the $2 \mathrm{~N}$ (sandpaper) object was lifted.

Force increases were not associated with impaired capacity to modulate fingertip forces smoothly, contrary to anecdotal data
(Cole, 1991; Kinoshita and Francis, 1966). Likewise, we found no evidence that older adults were less able than young adults to program their fingertip forces on the basis of sensorimotor memory of the object weight obtained from the preceding lift (Kinoshita and Francis, 1996). However, this "anticipatory control" policy (Johansson and Cole, 1994) operates over a longer time scale (across trials) as compared with the on-line scaling of motor commands for visually guided reaches to objects. During reaching, feedforward controllers reportedly are impaired in older adults (Warabi et al., 1986; Pohl et al., 1996), although a conflicting report exists for reaches to moving objects (Carnahan et al., 1998).

We theorize that the fingertip forces of adults older than 60 years may have increased in the Friction experiment to compensate for impoverished encoding of friction by cutaneous mechanoreceptive afferents. Adapting one's "central set" in this manner would seem reasonable for individuals who cannot rapidly adjust fingertip forces to new surfaces (see Johansson, 1996), particularly when objects of varying frictional properties may be encountered. Likewise, the older subjects' default scaling of force trajectories for the most slippery surface (acetate) may be a strategic compensation for marginally effective mechanisms that adjust force for friction on-line. This affords greater grip/load ratios throughout the loading phase and will reduce the number and magnitude of slips. Slips commonly occur in young adults when grip surfaces are randomly varied between nonslippery and slippery materials, which may explain why some young adults also used a default force scaling when grip surface could not be anticipated (Johansson and Westling, 1984; Johansson, 1996).

The fingertip force response latencies observed for young sub- 
jects $(\sim 100 \mathrm{msec})$ when new surface frictions were encountered are consistent with results from previous studies (Johansson and Westling, 1984; Edin et al., 1992; Jenmalm and Johansson, 1997). The latencies that older subjects demonstrated ( $200 \mathrm{msec})$ represent substantial delays, considering that the loading phase lasted only $\sim 300 \mathrm{msec}$. However, the relatively long latencies observed for our old subjects should be viewed cautiously, especially for S-A trials, given the tendency for these subjects to scale the initial portion of their grip force trajectories always for the more slippery surface. Nevertheless, we were particularly interested in A-S trials because all subjects used force trajectories at the beginning of their loading phases during A-S trials that were scaled for the preceding acetate trial. This provided more force than was needed to lift the sandpaper object without slip, regardless of age. Therefore, from the perspective of avoiding slip there was no urgency to reduce grip force in either group of subjects, yet young subjects began slowing their rate of grip force rise $\sim 100$ msec earlier than did old subjects.

We view our results as consistent with a deteriorating capacity to encode frictional information in the discharges of cutaneous mechanoreceptive afferents. Friction at skin-object contact patches is encoded nearly exclusively by fast-adapting afferents with small receptive fields (FA I; Johansson and Westling, 1987), with Meissner's corpuscles as the putative endings (Vallbo and Johansson, 1984). In old age, Meissner's corpuscles decrease substantially in number, and their morphology changes (Dickens et al., 1963; Cauna, 1965; Bolton et al., 1966). Tactile sensibility deficits in old age are consistent with these changes (Kenshalo, 1986; Gescheider et al., 1994; Stevens et al., 1998), as is microneurographic evidence of diminished efficiency for mechanoelectric transduction (Schmidt et al., 1990). Additionally, tactile deficits may increase in old age from reduced skin hydration and resultant changes in skin mechanics. Perception of roughness becomes less sensitive as skin hydration decreases (Verrillo et al., 1998), which further may explain our observation in older adults of disproportional increases in relative safety margin as skin slipperiness increases.

We attribute the lengthened response latencies that we observed to central delays, probably from delayed neural detection of friction. The latencies increased too much to result directly from age-related changes in mechanoelectric transduction or peripheral nerve conduction velocity. These latter measures show increases of a few to several percent in old age (Dorfman and Bosley, 1979; Schmidt et al., 1990; Caruso et al., 1993). The hypothesized central delays do not reflect the ubiquitous behavioral slowing that occurs in old age (Welford et al., 1969). The time taken to begin reducing grip force after object set-down did not increase with age, probably because the force impulse at set-down is encoded robustly by FA II afferents in the digits, palm, and wrist (Westling and Johansson, 1987).

It may appear paradoxical to attribute age-related changes of fingertip forces to deficiencies in processing FA I afferent discharges, when these force changes corresponded only weakly to pressure sensibility thresholds (Semmes-Weinstein). Although we observed statistically significant elevations in pressure thresholds in our old subjects (reported in Cole et al., 1998), these estimates seem likely to include substantial error inherent to the use of handheld filaments. Under controlled test conditions, psychophysical detection thresholds correspond well with mechanical thresholds for evoking FA I and FA II (fast-adapting large receptive field) discharges in digit afferents, with circumstantial evidence strongly favoring FA I afferents (Johansson and Vallbo,
1979; Vallbo and Johansson, 1984). However, detection thresholds vary substantially with the exact placement of the probe, most likely in relation to the location of fast-adapting afferents (Johansson and Vallbo, 1979). Such sampling errors may become large in older subjects, given the loss of Meissner's corpuscles.

Recently, we failed to support the theory that diminished cutaneous sensory functioning in old age contributes to the agerelated increase in the time needed to grasp and lift a small smooth sphere (Cole et al., 1998). In that study older adults did not require inordinately longer time to complete the task blindfolded as compared with young subjects. However, the sphere did not vary in its mechanical properties or location, yielding a task that may be relatively insensitive to deteriorating FA I function. The cutaneous information needed to locate the object and position the digits for grasp may be encoded sufficiently by SA I (slowing adapting) afferents (Johansson and Westling, 1987; Westling and Johansson, 1987). These afferents are not lost in old age, assuming that Merkel-neurite complexes comprise the receptor (Cauna, 1965; Vallbo and Johansson, 1984).

Our earlier findings (Cole et al., 1998) and the present results indicate that functional motor deficits from tactile sensory impairments in old age will vary with task and behavioral context. Greater deficits should occur for tasks critically dependent on fingertip events that fast-adapting afferents preferentially encode (e.g., handling objects that vary in friction, restraining objects subjected to unexpected external loading; see Johansson, 1996) and in contexts when anticipatory control of fingertip force becomes unreliable.

\section{REFERENCES}

Bolton CF, Winkelmann RK, Dyck MD (1966) A quantitative study of Meissner's corpuscles in man. Neurology 16:1-9.

Cadoret G, Smith A (1996) Friction, not texture, dictates grip forces used during object manipulation. J Neurophysiol 75:1963-1969.

Carnahan H, Vandervoort AA, Swanson LR (1998) The influence of aging and target motion on the control of prehension. Exp Aging Res 24:289-306.

Caruso G, Nilsson J, Crisci C, Nolano M, Massini R, Lullo F (1993) Sensory nerve findings by tactile stimulation of median and ulnar nerves in healthy subjects of different ages. Electroencephalogr Clin Neurophysiol 89:392-398.

Cauna N (1965) The effects of aging on the receptor organs of the human dermis. In: Advances in biology of the skin, Vol VI (Montagna W, ed), pp 63-96. New York: Pergamon.

Cole KJ (1991) Grasp force control in older adults. J Mot Behav 23:251-258.

Cole KJ, Beck CL (1994) The stability of precision grip force in older adults. J Mot Behav 26:171-177.

Cole KJ, Rotella DL, Harper JG (1998) Tactile impairments cannot explain the effect of age on a grasp and lift task. Exp Brain Res 121:263-269.

Dickens WN, Winkelmann RK, Mulder DW (1963) Cholinesterase demonstration of dermal nerve endings in patients with impaired sensation: a clinical and pathological study of 41 patients and 27 control subjects. Neurology 13:91-100.

Dorfman LJ, Bosley TM (1979) Age-related changes in peripheral and central nerve conduction in man. Neurology 29:38-44.

Edin BB, Westling G, Johansson RS (1992) Independent control of human finger-tip forces at individual digits during precision lifting. J Physiol (Lond) 450:547-564.

Eliasson A, Gordon AM, Forssberg H (1995) Tactile control of isometric fingertip forces during grasping in children with cerebral palsy. Dev Med Child Neurol 37:72-84.

Falconer J, Hughes SL, Naughton BJ, Singer R, Chang RW, Sinacore JM (1991) Self report and performance-based hand function tests as correlates of dependency in the elderly. J Am Geriatr Soc 39:695-699.

Folstein MF, Folstein SE, McHugh PR (1975) Mini-mental state: a practical method for grading the cognitive state of patients for the clinician. J Psychiatr Res 12:189-198. 
Gescheider GA, Bolanowski SJ, Hall KL, Hoffman KE, Verrillo RT (1994) The effects of aging on information-processing channels in the sense of touch. I. Absolute sensitivity. Somatosens Mot Res 11:345-357.

Hackel ME, Wolfe GA, Bang SM, Canfield JS (1992) Changes in hand function in the aging adult as determined by the Jebsen test of hand function. Phys Ther 72:373-377.

Jebsen RH, Taylor N, Trieschmann RB, Trotter MJ, Howard LA (1969) An objective and standardized test of hand function. Arch Phys Med Rehabil 50:311-319.

Jenmalm P, Johansson RS (1997) Visual and somatosensory information about object shape control manipulative fingertip forces. J Neurosci 17:4486-4499.

Jette AM, Branch LG, Berlin J (1990) Musculoskeletal impairments and physical disablement among the aged. J Gerontol A Biol Sci Med Sci 45:M203-M208.

Johansson RS (1996) Sensory control of dexterous manipulation in humans. In: Hand and brain (Wing A, Haggard P, Flanagan J, eds), pp 381-414. San Diego: Academic.

Johansson RS, Cole KJ (1994) Grasp stability during manipulative actions. Can J Physiol Pharmacol 72:511-524.

Johansson RS, Vallbo AB (1979) Detection of tactile stimuli. Threshold of afferent units related to psychophysical thresholds in the human hand. J Physiol (Lond) 297:405-422.

Johansson RS, Westling G (1984) Roles of glabrous skin receptors and sensorimotor memory in automatic control of precision grip when lifting rougher or more slippery objects. Exp Brain Res 56:550-554.

Johansson RS, Westling G (1987) Signals in tactile afferents from the fingers eliciting adaptive motor responses during precision grip. Exp Brain Res 66:141-154.

Johansson RS, Westling G (1991) Afferent signals during manipulative tasks in humans. In: Information processing in the somatosensory system (Franzen O, Westman J, eds), pp 25-48. London: MacMillan.

Kawai S, Kinoshita H, Ikuta K (1995) Effects of varied surface conditions on regulation of grip force during holding tasks using a precision grip [Japanese text, English abstract]. Jpn J Phys Fitness Sport Med 40:519-530.

Keen DA, Yue GH, Enoka RM (1994) Training-related enhancement in the control of motor output in elderly humans. J Appl Physiol 77:2648-2658.

Kenshalo DR (1986) Somesthetic sensitivity in young and elderly humans. J Gerontol 41:732-742.

Kinoshita H, Francis PR (1996) A comparison of prehension force control in young and elderly individuals. Eur J Appl Physiol 74:450-460.
Kinoshita H, Backstrom L, Flanagan JR, Johansson RS (1997) Tangential torque effects on the control of grip forces when holding objects with a precision grip. J Neurophysiol 78:1619-1630.

Lindeman RH, Merenda PF, Gold R (1980) Introduction to bivariate and multivariate analysis. New York: Scott, Foresman.

Morgan M, Phillips JG, Bradshaw JL, Mattingley JB, Iansek R, Bradshaw JA (1994) Age-related motor slowness-simply strategic. J Gerontol A Biol Sci Med Sci 49:M133-M139.

Pacaud S, Welford AT (1989) Performance in relation to age and educational level: a monumental research. Exp Aging Res 15:123-136.

Pohl PS, Winstein CJ, Fisher BE (1996) The locus of age-related movement slowing: sensory processing in continuous goal-directed aiming. $\mathrm{J}$ Gerontol B Psychol Sci Soc Sci 51:94-102.

Potts RO, Buras E, Chrisman DA (1984) Changes with age in the moisture content of human skin. J Invest Dermatol 82:97-100.

Potvin AR, Syndulko K, Tourtellotte WW, Lemmon JA, Potvin JH (1980) Human neurologic function and the aging process. J Am Geriatr Soc 28:1-9.

Schmidt RF, Wahren LK, Hagbarth KE (1990) Multiunit neural responses to strong finger pulp vibration. I. Relationship to age. Acta Physiol Scand 140:1-10.

Stevens JC, Cruz LA, Marks LE, Lakatos S (1998) A multimodal assessment of sensory thresholds in aging. J Gerontol B Psychol Sci Soc Sci 53:263-272.

Vallbo AB, Johansson RS (1984) Properties of cutaneous mechanoreceptors in the human hand related to touch sensation. Hum Neurobiol 3:3-14.

Verrillo RT, Bolanowski SJ, Checkosky CM, McGlone FP (1998) Effects of hydration on tactile sensation. Somatosens Mot Res 15:93-108.

Warabi T, Noda H, Kato T (1986) Effect of aging on sensorimotor functions of eye and hand movements. Exp Neurol 92:686-697.

Welford AT, Norris AH, Shock NW (1969) Speed and accuracy of movement and their changes with age. Acta Psychol (Amst) 30:3-15.

Westling G, Johansson RS (1984) Factors influencing the force of control during precision grip. Exp Brain Res 53:277-284.

Westling G, Johansson RS (1987) Responses in glabrous skin mechanoreceptors during precision grip in humans. Exp Brain Res 66:128-140.

Williams ME, Hadler N, Earp JAL (1982) Manual ability as a marker of dependency in geriatric women. J Chronic Dis 35:115-122.

Yan JH, Thomas JR, Stelmach GE (1998) Aging and rapid aiming arm movement control. Exp Aging Res 24:155-168. 